\title{
Medical diagnosis in intuitionistic fuzzy context
}

\author{
Jeswin B. George ${ }^{1}$ and Shiny Jose ${ }^{2}$ \\ ${ }^{1}$ Department of Mathematics, BVM Holy Cross College \\ Cherpunkal, Kerala, India \\ e-mail: jeswinkomarathakunnel@gmail.com \\ ${ }^{2}$ Department of Mathematics, St. George College \\ Aruvithura, Kerala, India \\ e-mail: shinyjosedavis@gmail.com
}

Received: 2 January 2019

Revised: 13 April 2019

Accepted: 17 April 2019

\begin{abstract}
In this paper, we introduce a distance formula for intuitionistic fuzzy sets called novel tangent inverse distance and found that it satisfies the properties of a distance measure. Application of tangent inverse distance in medical diagnosis is also discussed.
\end{abstract}

Keywords: Intuitionistic fuzzy sets, Tangent inverse distance, Medical diagnosis.

2010 Mathematics Subject Classification: 03E72.

\section{Introduction}

Intuitionistic fuzzy sets (IFSs) were proposed by K. T. Atanassov [1, 2] as an extension of Zadeh's fuzzy sets. They can express and process uncertainty in a better way, using hesitation degree. Most of the applications of IFS are implemented using some distance measures based approach. In literature many distance measures have been defined between IFSs like Hamming distance, normalized Hamming distance, Euclidean distance, normalized Euclidean distance etc. Among them tangent inverse distance has more accuracy and perfection [4].

In this paper, we propose the simplest form for tangent inverse distance given in [4] and discuss its properties and application in the field of medical diagnosis (see also [3, 7-10]). 


\section{Preliminaries}

Definition 2.1. Intuitionistic fuzzy sets $[1,2]$. Let $X$ be a given set. An intuitionistic fuzzy set $A$ in $X$ is given by $A=\left\{\left(x, \mu_{A}(x), v_{A}(x)\right) \mid x \in X\right\}$ where $\mu_{A}, v_{A}: X \rightarrow[0,1], \mu_{A}(x)$ is the degree of membership of the element $x$ in $A$ and $v_{A}(x)$ is the degree of non-membership of $x$ in $A$, and $0 \leq \mu_{A}(x)+v_{A}(x) \leq 1$. For each $x \in X, \pi_{A}(x)=1-\mu_{A}(x)-v_{A}(x)$ is the degree of hesitation.

Definition 2.2. Hamming distance [7, 8]. The Hamming distance between two intuitionistic fuzzy sets $A=\left(\mu_{A}\left(x_{i}\right), v_{A}\left(x_{i}\right), \pi_{A}\left(x_{i}\right)\right)$ and $B=\left(\mu_{B}\left(x_{i}\right), v_{B}\left(x_{i}\right), \pi_{B}\left(x_{i}\right)\right)$ is given by

$$
d_{I F S}^{1}(A, B)=\frac{1}{2} \sum_{i=1}^{n}\left(\left|\mu_{A}\left(x_{i}\right)-\mu_{B}\left(x_{i}\right)\right|+\left|v_{A}\left(x_{i}\right)-v_{B}\left(x_{i}\right)\right|+\left|\pi_{A}\left(x_{i}\right)-\pi_{B}\left(x_{i}\right)\right|\right) .
$$

Definition 2.3. Normalized Hamming distance [7, 8]. The normalized Hamming distance between two intuitionistic fuzzy sets $A=\left(\mu_{A}\left(x_{i}\right), v_{A}\left(x_{i}\right), \pi_{A}\left(x_{i}\right)\right)$ and $B=\left(\mu_{B}\left(x_{i}\right), v_{B}\left(x_{i}\right), \pi_{B}\left(x_{i}\right)\right)$ is given by

$$
l_{I F S}^{1}(A, B)=\frac{1}{2 n} \sum_{i=1}^{n}\left(\left|\mu_{A}\left(x_{i}\right)-\mu_{B}\left(x_{i}\right)\right|+\left|v_{A}\left(x_{i}\right)-v_{B}\left(x_{i}\right)\right|+\left|\pi_{A}\left(x_{i}\right)-\pi_{B}\left(x_{i}\right)\right|\right) .
$$

Definition 2.4. Tangent inverse distance [4]. Let $A=\left(\mu_{A}\left(x_{i}\right), v_{A}\left(x_{i}\right), \pi_{A}\left(x_{i}\right)\right)$ and $B=$ $\left(\mu_{B}\left(x_{i}\right), v_{B}\left(x_{i}\right), \pi_{B}\left(x_{i}\right)\right)$ be two intuitionistic fuzzy sets. Then the tangent inverse distance is defined as

$$
\begin{gathered}
\operatorname{TID}_{I F S}(A, B)=\frac{1}{2(n+1)} \sum_{i=1}^{n}\left[\operatorname { t a n } ^ { - 1 } \left[\frac { \pi } { 4 } \left[1+\left|\mu_{A}\left(x_{i}\right)-\mu_{B}\left(x_{i}\right)\right|+\left|v_{A}\left(x_{i}\right)-v_{B}\left(x_{i}\right)\right|+\right.\right.\right. \\
\left.\left.\left.\left|\pi_{A}\left(x_{i}\right)-\pi_{B}\left(x_{i}\right)\right|\right]\right]\right]
\end{gathered}
$$

Propositions 2.5. [4].

(i) $\quad T I D_{I F S}(A, B)>0$,

(ii) $\quad T I D_{I F S}(A, B)=T I D_{I F S}(B, A)$,

(iii) If $A \subseteq B \subseteq C$ then $T I D_{I F S}(A, C) \geq T I D_{I F S}(A, B) \& T I D_{I F S}(A, C) \geq T T_{I F S}(B, C)$.

\section{A novel tangent inverse distance formula}

Definition 3.1. Novel tangent inverse distance. Let $A=\left(\mu_{A}(x), v_{A}(x), \pi_{A}(x)\right)$ and $B=$ $\left(\mu_{B}(x), v_{B}(x), \pi_{B}(x)\right)$ be two intuitionistic fuzzy sets. Then the tangent inverse distance is defined as

$$
\operatorname{TID}_{I F S}(A, B)=\frac{1}{2(n+1)} \sum_{i=1}^{n}\left[\tan ^{-1}\left[d_{i}\right]\right]
$$

where

$$
d_{i}=\left|\mu_{A}\left(x_{i}\right)-\mu_{B}\left(x_{i}\right)\right|+\left|v_{A}\left(x_{i}\right)-v_{B}\left(x_{i}\right)\right|+\left|\pi_{A}\left(x_{i}\right)-\pi_{B}\left(x_{i}\right)\right|
$$


for $i=1,2, \ldots, n$.

\section{Propositions 3.2.}

(i) $\quad T I D_{I F S}(A, B)>0$,

(ii) $T I D_{I F S}(A, B)=T I D_{I F S}(B, A)$,

(iii) If $A \subseteq B \subseteq C$ then $T I D_{I F S}(A, C) \geq T I D_{I F S}(A, B) \& T I D_{I F S}(A, C) \geq T T_{I F S}(B, C)$.

Proof.

(i) Since $\left|\mu_{A}\left(x_{i}\right)-\mu_{B}\left(x_{i}\right)\right|,\left|v_{A}\left(x_{i}\right)-v_{B}\left(x_{i}\right)\right|,\left|\pi_{A}\left(x_{i}\right)-\pi_{B}\left(x_{i}\right)\right| \geq 0$ we get $d_{i} \geq 0 \forall i=$ $1,2, \ldots, n$.

Therefore, $\tan ^{-1}\left[d_{i}\right] \geq 0 \quad \forall i=1,2, \ldots, n$.

Therefore, $\sum_{i=1}^{n}\left[\tan ^{-1}\left[d_{i}\right]\right] \geq 0$

Therefore, $\frac{1}{2(n+1)} \sum_{i=1}^{n}\left[\tan ^{-1}\left[d_{i}\right]\right] \geq 0$

i.e., $\operatorname{TID}_{I F S}(A, B)>0$

(ii) $\operatorname{TID}_{I F S}(A, B)=\frac{1}{2(n+1)} \sum_{i=1}^{n}\left[\tan ^{-1}\left[d_{i}\right]\right]$

$$
\begin{aligned}
& =\frac{1}{2(n+1)} \sum_{i=1}^{n}\left[\tan ^{-1}\left[\left|\mu_{A}\left(x_{i}\right)-\mu_{B}\left(x_{i}\right)\right|+\left|v_{A}\left(x_{i}\right)-v_{B}\left(x_{i}\right)\right|+\left|\pi_{A}\left(x_{i}\right)-\pi_{B}\left(x_{i}\right)\right|\right]\right] \\
& =\frac{1}{2(n+1)} \sum_{i=1}^{n}\left[\tan ^{-1}\left[\left|\mu_{B}\left(x_{i}\right)-\mu_{A}\left(x_{i}\right)\right|+\left|v_{B}\left(x_{i}\right)-v_{A}\left(x_{i}\right)\right|+\left|\pi_{B}\left(x_{i}\right)-\pi_{A}\left(x_{i}\right)\right|\right]\right] \\
& =\operatorname{TID}_{I F S}(B, A)
\end{aligned}
$$

(iii) We have if $A \subseteq B \subseteq C$ then $\left\{\begin{array}{l}\mu_{A}\left(x_{i}\right) \leq \mu_{B}\left(x_{i}\right) \leq \mu_{C}\left(x_{i}\right) \\ v_{A}\left(x_{i}\right) \geq v_{B}\left(x_{i}\right) \geq v_{C}\left(x_{i}\right) \\ \pi_{A}\left(x_{i}\right) \geq \pi_{B}\left(x_{i}\right) \geq \pi_{C}\left(x_{i}\right)\end{array}\right.$

So,

$$
\begin{aligned}
\left|\mu_{A}\left(x_{i}\right)-\mu_{B}\left(x_{i}\right)\right| & \leq\left|\mu_{A}\left(x_{i}\right)-\mu_{C}\left(x_{i}\right)\right| \\
\left|\mu_{B}\left(x_{i}\right)-\mu_{C}\left(x_{i}\right)\right| & \leq\left|\mu_{A}\left(x_{i}\right)-\mu_{C}\left(x_{i}\right)\right| \\
\left|v_{A}\left(x_{i}\right)-v_{B}\left(x_{i}\right)\right| & \leq\left|v_{A}\left(x_{i}\right)-v_{C}\left(x_{i}\right)\right| \\
\left|v_{B}\left(x_{i}\right)-v_{C}\left(x_{i}\right)\right| & \leq\left|v_{A}\left(x_{i}\right)-v_{C}\left(x_{i}\right)\right| \\
\left|\pi_{A}\left(x_{i}\right)-\pi_{B}\left(x_{i}\right)\right| & \leq\left|\pi_{A}\left(x_{i}\right)-\pi_{C}\left(x_{i}\right)\right| \\
\left|\pi_{B}\left(x_{i}\right)-\pi_{C}\left(x_{i}\right)\right| & \leq\left|\pi_{A}\left(x_{i}\right)-\pi_{C}\left(x_{i}\right)\right|
\end{aligned}
$$

From this we get $\operatorname{TID}_{I F S}(A, C) \geq \operatorname{TID}_{I F S}(A, B) \& T I D_{I F S}(A, C) \geq \operatorname{TID}_{I F S}(B, C)$.

\section{Illustration}

Following the numerical example in [3], let us consider four patients $\left\{P_{1}, P_{2}, P_{3}, P_{4}\right\}$ and the set of symptoms $S=\left\{S_{1}\right.$ Temperature, $S_{2}$ Headache, $S_{3}$ Stomach pain, $S_{4}$ Cough, $S_{5}$ Chest pain $\}$. The intuitionistic fuzzy relation $Q(P \rightarrow S)$ is given as in Table 1 . Let the set of diseases $D=$ $\left\{D_{1}\right.$ Viral fever, $D_{2}$ Malaria, $D_{3}$ Typhoid, $D_{4}$ Stomach problem, $D_{5}$ Chest problem $\}$. The intuitionistic fuzzy relation $R(S \rightarrow D)$ is given as in Table 2). 


\begin{tabular}{|c|c|c|c|c|c|}
\hline$A$ & Temperature & Headache & Stomach pain & Cough & Chest pain \\
\hline$P_{1}$ & $(0.8,0.1,0.1)$ & $(0.6,0.1,0.3)$ & $(0.2,0.8,0.0)$ & $(0.6,0.1,0.3)$ & $(0.1,0.6,0.3)$ \\
\hline$P_{2}$ & $(0.0,0.8,0.2)$ & $(0.4,0.4,0.2)$ & $(0.6,0.1,0.3)$ & $(0.1,0.7,0.2)$ & $(0.1,0.8,0.1)$ \\
\hline$P_{3}$ & $(0.8,0.1,0.1)$ & $(0.8,0.1,0.1)$ & $(0.0,0.6,0.4)$ & $(0.2,0.7,0.1)$ & $(0.0,0.5,0.5)$ \\
\hline$P_{4}$ & $(0.6,0.1,0.3)$ & $(0.5,0.4,0.1)$ & $(0.3,0.4,0.3)$ & $(0.7,0.2,0.1)$ & $(0.3,0.4,0.3)$ \\
\hline
\end{tabular}

Table 1. Patient-Symptom Relation

\begin{tabular}{|c|c|c|c|c|c|}
\hline$B$ & Viral fever & Malaria & Typhoid & Stomach problem & Chest problem \\
\hline Temperature & $(0.4,0.0,0.6)$ & $(0.7,0.0,0.3)$ & $(0.3,0.3,0.4)$ & $(0.1,0.7,0.2)$ & $(0.1,0.8,0.1)$ \\
\hline Headache & $(0.3,0.5,0.2)$ & $(0.2,0.6,0.2)$ & $(0.6,0.1,0.3)$ & $(0.2,0.4,0.4)$ & $(0.0,0.8,0.2)$ \\
\hline Stomach pain & $(0.1,0.7,0.2)$ & $(0.0,0.9,0.1)$ & $(0.2,0.7,0.1)$ & $(0.8,0.0,0.2)$ & $(0.2,0.8,0.0)$ \\
\hline Cough & $(0.4,0.3,0.3)$ & $(0.7,0.0,0.3)$ & $(0.2,0.6,0.2)$ & $(0.2,0.7,0.1)$ & $(0.2,0.8,0.0)$ \\
\hline Chest Pain & $(0.1,0.7,0.2)$ & $(0.1,0.8,0.1)$ & $(0.1,0.9,0.0)$ & $(0.2,0.7,0.1)$ & $(0.8,0.1,0.1)$ \\
\hline
\end{tabular}

Table 2. Symptom-Disease Relation

Using distance formula given in Definition 3.1 we get the following table.

\begin{tabular}{|c|c|c|c|c|c|}
\hline $\mathrm{T}$ & Viral fever & Malaria & Typhoid & Stomach problem & Chest problem \\
\hline$P_{1}$ & 0.2015 & $\mathbf{0 . 1 7 2 6}$ & 0.1923 & 0.3244 & 0.3168 \\
\hline$P_{2}$ & 0.2464 & 0.2795 & 0.2164 & $\mathbf{0 . 1 1 2 7}$ & 0.2627 \\
\hline$P_{3}$ & 0.2638 & 0.2851 & $\mathbf{0 . 2 1 7 6}$ & 0.3019 & 0.3154 \\
\hline$P_{4}$ & $\mathbf{0 . 2 1 1 8}$ & 0.2148 & 0.2659 & 0.2939 & 0.3393 \\
\hline
\end{tabular}

Table 3. Novel tangent inverse distance

From the above table, $P_{1}$ is diagnosed with malaria, $P_{2}$ is diagnosed with stomach problem, $P_{3}$ is diagnosed with typhoid and $P_{4}$ is diagnosed with Viral fever.

Note: If the distance between a patient and a particular disease is the shortest, the patient is likely to have that disease.

\section{Conclusions}

We have introduced a novel distance formula for IFS called novel tangent inverse distance. It is elegant and simple than that given in [4]. It is useful to find the distance between IFS especially in medical diagnosis.

\section{References}

[1] Atanassov, K. T. (1986). Intuitionistic fuzzy sets, Fuzzy Sets and Systems, 20 (1), 87-96.

[2] Atanassov, K. T. (1989). More on Intuitionistic fuzzy sets, Fuzzy Sets and Systems, 33 (1), $37-45$. 
[3] De, S. K., Biswas, R., \& Roy, A. R. (2001). An application of intuitionistic fuzzy sets in medical diagnosis, Fuzzy Sets and Systems, 117 (2), 209-213.

[4] Edward Samuel, A. \& Narmadhagnanam, R. (2018). Intuitionistic Fuzzy Sets in Medical Diagnosis, International Journal of Mathematical Archive, 9 (1), 1-5.

[5] Ejegwa, P. A., Onoja, A. M., \& Emmanuel, I. T. (2014). A Note on Some Models of Intuitionistic Fuzzy Sets in Real Life Situations, Journal of Global Research in Mathematical Archives, 2 (5), 42-50.

[6] Intarapaiboon, P. (2014). New Similarity Measures for Intuitionistic Fuzzy Sets, Applied Mathematical Sciences, 8 (45), 39-50.

[7] Szmidt, E., \& Kacprzyk J. (2002). An intuitionistic fuzzy set based approach to intelligent data analysis: an application to medical diagnosis. Recent Advances in Intelligent Paradigms and Applications (A. Abraham, L. Jain, J. Kacprzyk, Eds), Springer, Berlin, 57-70.

[8] Szmidt, E., \& Kacprzyk J. (2005). A similarity measure for intuitionistic fuzzy sets and its application in supporting medical diagnostic reasoning. Lecture Notes in Artificial Intelligence, Vol. 3070, Springer, Berlin, 388-393.

[9] Todorova, L., Atanassov, K., Hadjitodorov, S., \& Vassilev, P. (2007). On an intuitionistic fuzzy approach for decision making in medicine: Part 1, Int. Journal Bioautomation, 6, 92-101.

[10] Todorova, L., Atanassov, K., Hadjitodorov, S., \& Vassilev, P. (2007). On an intuitionistic fuzzy approach for decision making in medicine. Part 2. Int. Journal Bioautomation, 7 , 64-69. 